\title{
Reduction of Oxygen-Carrying Capacity Weakens the Effects of Increased Plasma Viscosity on Cardiac Performance in Anesthetized Hemodilution Model
}

\author{
Surapong Chatpun ${ }^{1}$ and Pedro Cabrales ${ }^{2}$ \\ ${ }^{1}$ Institute of Biomedical Engineering, Faculty of Medicine, Prince of Songkla University, Songkhla 90110, Thailand \\ ${ }^{2}$ Department of Bioengineering, University of California, San Diego, La Jolla, CA 92093, USA
}

Correspondence should be addressed to Surapong Chatpun, schatpun@gmail.com

Received 30 April 2012; Accepted 4 June 2012

Academic Editors: J.-H. Baumert, A. Mizutani, and C. Motamed

Copyright (C) 2012 S. Chatpun and P. Cabrales. This is an open access article distributed under the Creative Commons Attribution License, which permits unrestricted use, distribution, and reproduction in any medium, provided the original work is properly cited.

\begin{abstract}
We investigated the effects of reduced oxygen-carrying capacity on cardiac function during acute hemodilution, while the plasma viscosity was increased in anesthetized animals. Two levels of oxygen-carrying capacity were created by 1-step and 2step hemodilution in male golden Syrian hamsters. In the 1-step hemodilution (1-HD), 40\% of the animals' blood volume (BV) was exchanged with $6 \%$ dextran $70 \mathrm{kDa}$ (Dx70) or dextran $2000 \mathrm{kDa}(\mathrm{Dx} 2 \mathrm{M})$. In the 2-step hemodilution (2-HD), 25\% of the animals' BV was exchanged with Dx70 followed by $40 \%$ BV exchanged with Dx70 or Dx2M after 30 minutes of first hemodilution. Oxygen delivery in the 2-HD group consequently decreased by $17 \%$ and $38 \%$ compared to that in the 1 -HD group hemodiluted with Dx70 and Dx2M, respectively. End-systolic pressure and maximum rate of pressure change in the 2-HD group significantly lowered compared with that in the 1-HD group for both Dx70 and Dx2M. Cardiac output in the 2-HD group hemodiluted with Dx2M was significantly higher compared with that hemodiluted with Dx70. In conclusion, increasing plasma viscosity associated with lowering oxygen-carrying capacity should be considerably balanced to maintain the cardiac performance, especially in the state of anesthesia.
\end{abstract}

\section{Introduction}

Hemodilution reduces the circulating number of red blood cells (RBCs), decreases oxygen-carrying capacity, and lowers whole blood viscosity. To maintainf tissue oxygenation preventing multiorgan dysfunction, cardiac output (CO) increases to compensate for the reduction in oxygencarrying capacity. The acute normovolemic hemodilution in anesthetized dogs revealed that myocardial blood flow significantly led an increased preservation of both myocardial oxygen-carrying capacity and consumption $[1,2]$. Sufficient oxygen is normally offloaded from the hemoglobin in the RBCs unless the hematocrit (Hct) drops to very low values.

There is a trigger point which is necessary to readminister the RBCs into the circulation. The threshold for a blood transfusion is set at $\sim 7 \mathrm{~g}$ of hemoglobin per $100 \mathrm{~mL}$ of blood. However, the findings by Tsai and Intaglietta [3] indicated that hemoglobin concentration can be lowered to $6 \mathrm{~g} / \mathrm{dL}(18 \% \mathrm{Hct})$ if the blood viscosity at a physiological level is maintained with a high viscosity plasma expander (HVPE). Other studies using an awake animal model as well as an anesthetized animal model have shown that microvascular function is impaired if the blood viscosity is severely decreased by hemodilution rather than an insufficient oxygen-carrying capacity [4-8]. A recent study by Chatpun and Cabrales [9] found that dextran $2000 \mathrm{kDa}$ with a viscosity of $6.3 \mathrm{cP}$ provides positive effects on cardiac performance by reducing systemic vascular resistance compared to dextran $2000 \mathrm{kDa}$ with a viscosity of $2.2 \mathrm{cP}$, using a moderate hemodilution $(28 \% \mathrm{Hct})$ in anesthetized hamsters.

Although many studies in awake animals have demonstrated that severe hemodilution with an HVPE provides beneficial effects on microvascular function and perfusion 
compared to a lower viscosity plasma expander, there might be different outcomes when applying this level of hemodilution in anesthetized animals, especially to vital organ function such as heart and brain function. Furthermore, there are few studies about the effects of plasma viscosity and oxygen-carrying capacity on cardiac function in anesthetized animals $[10,11]$. Only the effects of the degree of anemia using different exchange solutions on cardiac function have been elucidated [1, 12-15].

This study aims to investigate the effects of increased plasma viscosity with a reduction of oxygen-carrying capacity by a different degree of hemodilution on cardiac function. Two degrees of isovolemic hemodilution were performed by the 1-step hemodilution and 2-step hemodilution to reduce the Hcts to $28 \%$ and $18 \%$, respectively. Dextran $70 \mathrm{kDa}$ and dextran $2000 \mathrm{kDa}$ were used as plasma expanders in these hemodilution models. Left ventricular cardiac function indices were derived from the pressure-volume (PV) measurement using a miniaturized PV conductance catheter. Systemic parameters such as mean arterial pressure and heart rate were also monitored and evaluated in this study.

\section{Materials and Methods}

2.1. Animal Preparation. This study was experimentally performed in anesthetized male Golden Syrian hamsters (Charles River Laboratories; Boston, MA) weighing 60$70 \mathrm{~g}$. Animal handling and care was conducted accordingly to the National Institutes of Health guidelines for the Care and Use of Laboratory Animals. The experimental protocol was approved by the institutional animal care committee. Surgery was performed following intraperitoneal (i.p.) injection of sodium pentobarbital $(50 \mathrm{mg} / \mathrm{kg})$. The left jugular vein was catheterized to allow plasma expander administration and the left femoral artery was cannulated for blood pressure monitoring and blood withdrawal and sampling. Furthermore, a tracheotomy was performed and cannulated with a polyethylene-90 tube to facilitate the animals' breathing. Animals were placed in the supine position on a heating pad to maintain the body temperature at $37^{\circ} \mathrm{C}$. Animals were subcutaneously administered $0.05-$ $0.10 \mathrm{mg} / \mathrm{kg}$ buprenorphine after surgery for analgesia. During the experiment, the state of anesthesia was checked by the animal's response to a toe pinching. A small bolus of sodium pentobarbital (10-15 mg/kg, i.p.) was given when a response was observed.

2.2. Inclusion Criteria. Animals under anesthesia were suitable for the experiment if the animals had no bleeding and systemic parameters were within normal range by (i) mean blood arterial pressure (MAP) above $80 \mathrm{mmHg}$, (ii) heart rate (HR) above 320 beats/minute and (iii) systemic hematocrit (Hct) above $45 \%$.

2.3. Systemic Parameters. The MAP was monitored continuously (MP150, Biopac System Inc.; Santa Barbara, CA, USA), except during blood exchange. The Hct was determined from centrifuged arterial blood samples taken in heparinized capillary tubes.

2.4. Rheological Properties. Viscosity was measured at a shear rate of $1601 / \mathrm{sec}$ at $37^{\circ} \mathrm{C}$ (Brookfield Engineering Laboratories; Middleboro, MA, USA). Colloid osmotic pressure (COP) of the plasma expanders and blood plasma were determined using a membrane colloid osmometer (Model 4420, Wescor Inc., Logan, UT, USA).

\subsection{Isovolemic Hemodilution Protocols}

2.5.1. Protocol 1:1-Step Hemodilution (1HD). Anesthetized hamsters had $40 \%$ of the estimated blood volume (BV) exchanged with the test solutions, dextran $2000 \mathrm{kDa}(\mathrm{Dx} 2 \mathrm{M})$, and dextran $70 \mathrm{kDa}$ (Dx70), lowering systemic Hct by $45 \%$.

2.5.2. Protocol 2: 2-Step Hemodilution (2HD). Anesthetized hamsters had $25 \%$ of the BV exchanged firstly with Dx70 followed by $40 \%$ of the BV exchanged with test solutions, Dx2M and Dx70, 30 minutes after hemodilution with Dx70. This protocol reduced the systemic Hct to $\sim 17-21 \%$.

Total BV was approximated as $7 \%$ of body weight. Test solutions were administered into the left jugular vein catheter at a rate of $100 \mu \mathrm{L} / \mathrm{min}$ with a simultaneous blood withdrawal at the same rate from the left femoral artery catheter by using a dual syringe pump (33 syringe pump, Harvard Apparatus, Holliston, MA, USA). Blood samples were collected at the end of the experiment for subsequent measurement of viscosity, plasma colloid osmotic pressure, and blood conductance. Animals were monitored for 60 minutes after completion of the hemodilution. Systemic parameters (MAP and Hct) were recorded and analyzed at baseline and 60 minutes after hemodilution. Figure 1 illustrates the study protocols.

2.6. Test Solutions. Two solutions were as follows: (1) $6 \%$ Dextran T2000 (Pharmacosmos, Holbaek, Denmark) in $0.9 \%$ sodium chloride $(\mathrm{NaCl})$ mixed with $10 \%$ human serum albumin and (2) $6 \%$ Dextran 70 in $0.9 \% \mathrm{NaCl}$ (B. Braun Medical, Irvine, CA, USA). The solution of dextran $2000 \mathrm{kDa}$ had a $6.3 \mathrm{cP}$ in viscosity and $43 \mathrm{mmHg}$ in COP while the solution of dextran $70 \mathrm{kDa}$ had a $3.0 \mathrm{cP}$ in viscosity and $52 \mathrm{mmHg}$ in COP.

2.7. Experimental Groups. Animals were randomly divided into two groups for each protocol. The first experimental group (high-viscosity group) was hemodiluted with $6 \%$ dextran $2000 \mathrm{kDa}$ (a group labeled Dx2M). The second experimental group (low-viscosity group) was hemodiluted with $6 \%$ dextran $70 \mathrm{kDa}$ (a group labeled Dx70).

2.8. Calculation of Oxygen Delivery. The total oxygen delivery to organs or tissues was calculated using the following equation:

$$
\begin{aligned}
\mathrm{O}_{2} \text { delivery }= & {\left[\left(\mathrm{RBC}_{\mathrm{Hb}} \times \gamma \times S_{A} \%\right)\right.} \\
& \left.+(1-\mathrm{Hct}) \times \alpha \times p \mathrm{O}_{2 A}\right] \times Q,
\end{aligned}
$$


1-step hemodilution
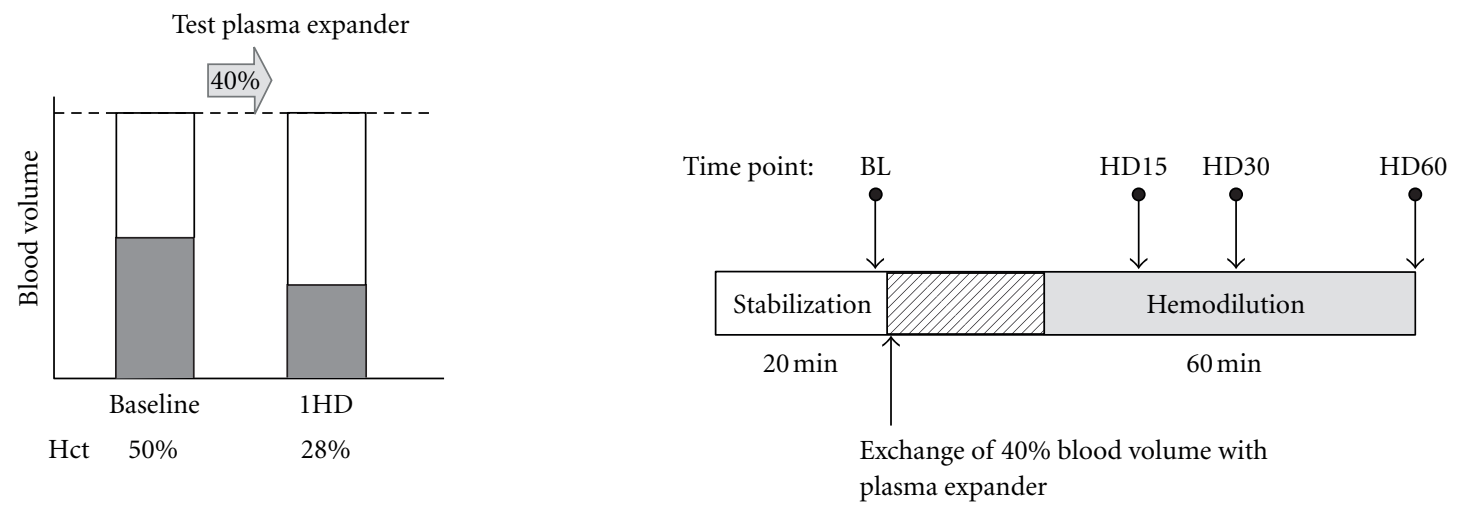

2-step hemodilution

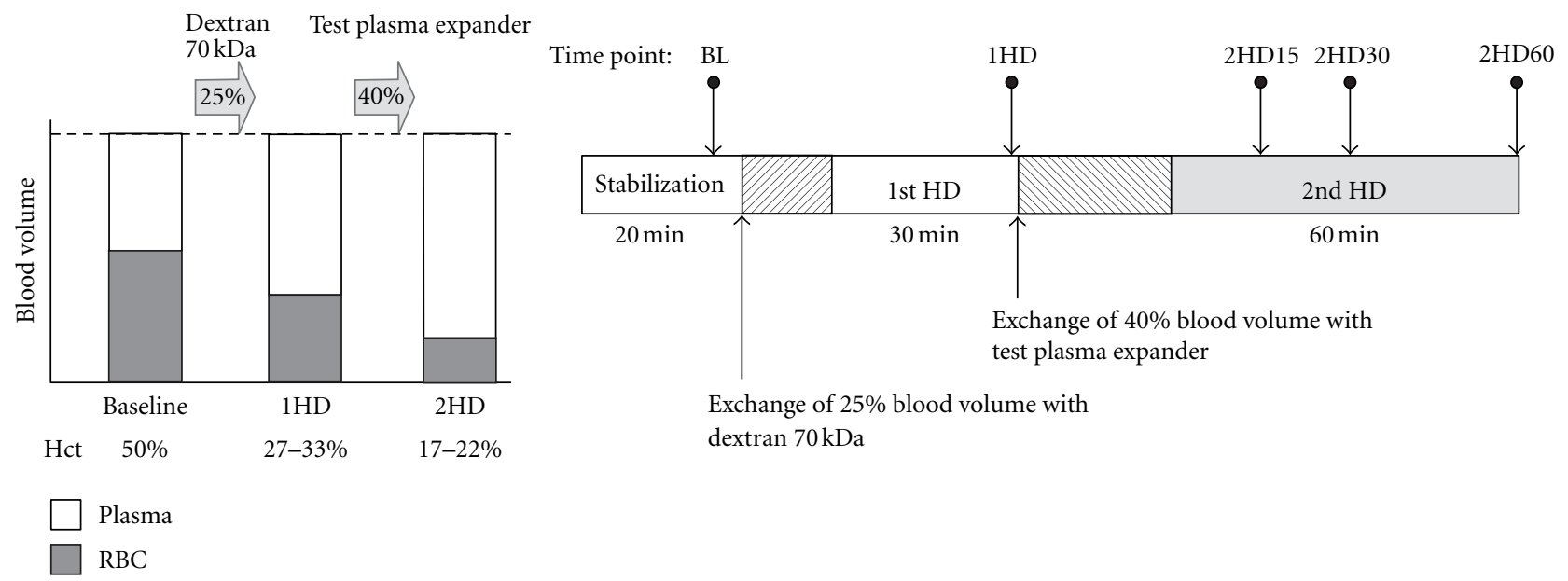

FIGURE 1: Schematic diagrams of acute isovolemic for the 1-step and 2-step hemodilution protocols. Hct, hematocrit; BL, baseline; HD, hemodilution.

where $\mathrm{RBC}_{\mathrm{Hb}}$ is the hemoglobin in RBCs (in grams of $\mathrm{Hb}$ per deciliter of blood); $\gamma$ is the oxygen-carrying capacity of saturated hemoglobin $(1.34 \mathrm{~mL} \mathrm{O} / \mathrm{gHb}) ; S_{A} \%$ is the arteriolar oxygen saturation; $(1-\mathrm{Hct})$ is the fractional plasma volume (in deciliter of plasma per deciliter of blood); $\alpha$ is the solubility of oxygen in plasma $\left(3.14 \times 10^{-3} \mathrm{~mL}\right.$ $\mathrm{O}_{2}$ /dLplasma $\mathrm{mmHg}$ ); $p \mathrm{O}_{2 A}$ is the arteriolar partial pressure of oxygen; $Q$ is the CO. The arteriolar oxygen saturation for hamster RBCs have been published previously [16].

2.9. Cardiac Function. Closed chest method was performed to assess cardiac function in this study [17]. The right common carotid artery was exposed allowing a $1.4 \mathrm{~F}$ pressurevolume conductance catheter (PV catheter; SPR-839, Millar Instruments, TX, USA) to be inserted. The PV catheter was advanced passing through the aortic valve into the left ventricle. At the baseline and at the end of the experiment, a bolus of $15 \%$ hypertonic saline $(10 \mu \mathrm{L})$ was intravenously injected to determine the parallel volume [18]. The pressure and volume signals were digitally acquired (MPVS300, Millar Instruments, Houston, TX, USA, and PowerLab 8/30, ADInstruments, Colorado Springs, CO, USA). Volume of the left ventricle was measured continuously in a conductance unit (relative volume unit, RVU) and converted to actual blood volume $(\mu \mathrm{L})$ at the end of the experiment using a known-volume cuvette calibration [10, 17]. Previous studies have shown that stroke volume measured by a conductance catheter was in agreement with the measurement using an ultrasound flow probe and an electromagnetic flow probe $[19,20]$. Cardiac function data were analyzed with PVAN software (version 3.6, Millar Instruments, TX, USA). The cardiac function indices were averaged from selected 8-12 cardiac cycles at each time point of interest.

2.10. Systemic Vascular Resistance. Systemic vascular resistance (SVR), the resistance to flow in the peripheral circulation, was approximately calculated from the measured values as $\mathrm{SVR}=\mathrm{MAP} / \mathrm{CO}$, where MAP is the mean arterial pressure and $\mathrm{CO}$ is cardiac output.

2.11. Statistical Analysis. Results are presented as mean \pm standard deviation (SD) unless otherwise addressed. Data between interested time points in a same group were analyzed using analysis of variance (ANOVA) and followed 
by post hoc analyses with the Dunnett's multiple comparison tests. An unpaired $t$-test with two-tailed was performed to compare between groups at the time point of interest. All statistics were calculated using GraphPad Prism 4.01 (GraphPad Software, San Diego, CA, USA). Results were considered statistically significant when $P<0.05$.

\section{Results}

All twenty-two animals under anesthesia tolerated without adverse conditions for the entire period of the experiment. The animals were assigned randomly into four experimental groups: 1HD-Dx70 $(n=6), 1 \mathrm{HD}-\mathrm{Dx} 2 \mathrm{M}(n=6), 2 \mathrm{HD}$ $\operatorname{Dx} 70(n=5)$, and 2HD-Dx2M $(n=5)$. The measured parameters at baseline were not statistically significant difference between the groups.

3.1. Systemic and Rheological Parameters. The changes of MAP after 1-step and 2-step hemodilution are demonstrated in Figure 2. Hemodilution markedly reduced MAP in both protocols. MAP in the $1 \mathrm{HD}-\mathrm{Dx} 2 \mathrm{M}$ group decreased to $84 \pm 6 \%$ of baseline while MAP in the 1HD-Dx70 group dropped to $81 \pm 2 \%$ of baseline. However, a further step in blood dilution caused a decrease of MAP to $71 \pm 9 \%$ and $68 \pm 2 \%$ of baseline in the 2HD-Dx70 and 2HD-Dx2M groups. Figure 3 shows that HR in each group decreased over time after hemodilution in both protocols. Changes in $\mathrm{Hct}, \mathrm{Hb}$, and blood and plasma viscosities were statistically different from baseline as presented in Table 1. Plasma viscosity in both protocols after hemodilution with Dx2M was significantly higher than that after hemodilution with Dx70 as well as whole blood viscosity.

3.2. Oxygen Delivery. Calculated values of oxygen delivery at the end of the experiment were compared to that at baseline. The oxygen delivery relative to baseline in the 2-step hemodilution with Dx70 decreased 17\% compared to that in the 1-step hemodilution with Dx70, whereas it was 38\% in the group hemodiluted with Dx2M as shown in Figure 4.

3.3. Cardiac Function. Cardiac function indices measured and derived from the pressure-volume measurement for the 1-step and 2-step hemodilutions are presented in Table 2. End-systolic pressure $\left(P_{\mathrm{es}}\right)$ significantly decreased compared with baseline $(P<0.05)$ while end-diastolic pressure $\left(P_{\text {ed }}\right)$ was unchanged from baseline in both protocols. An increase of plasma viscosity with Dx $2 \mathrm{M}$ decreased $P_{\text {es }}$ about $11 \%$ from baseline in the 1-step hemodilution, whereas it was $20 \%$ in the 2-step hemodilution. Dx70 decreased $P_{\text {es }}$ about $14 \%$ and $26 \%$ from baseline in the 1-step and 2-step hemodilutions. The peak positive time derivative of the left ventricular pressure $\left(d P / d t_{\max }\right)$ gradually decreased over time in the 1step hemodilution with Dx70, whereas it was maintained at the baseline level in the 1-step hemodilution with Dx2M. On the other hand, the peak negative time derivative of left ventricular pressure $\left(d P / d t_{\min }\right)$ in the 2-step hemodilution significantly decreased over time in both Dx70 and Dx2M groups $(P<0.05)$. With reduction of the hematocrit, from

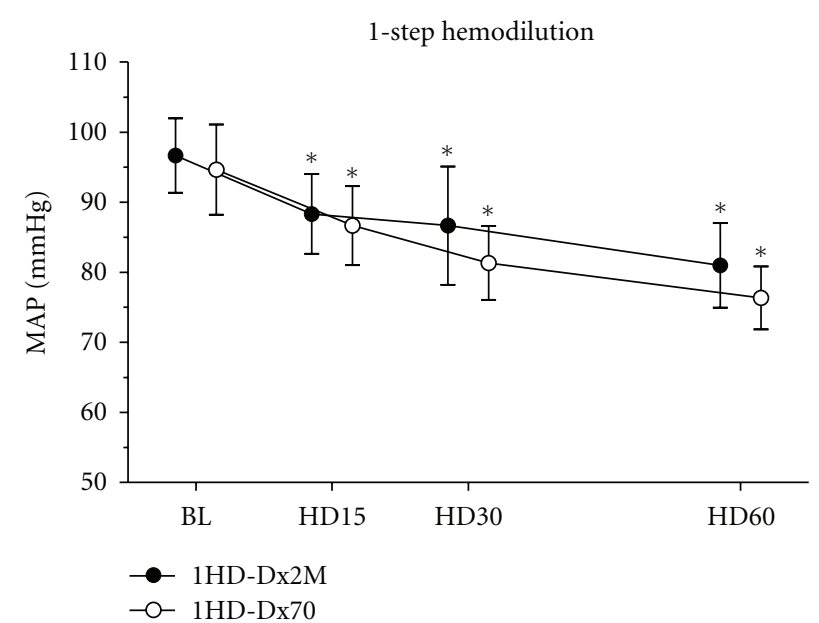

(a)

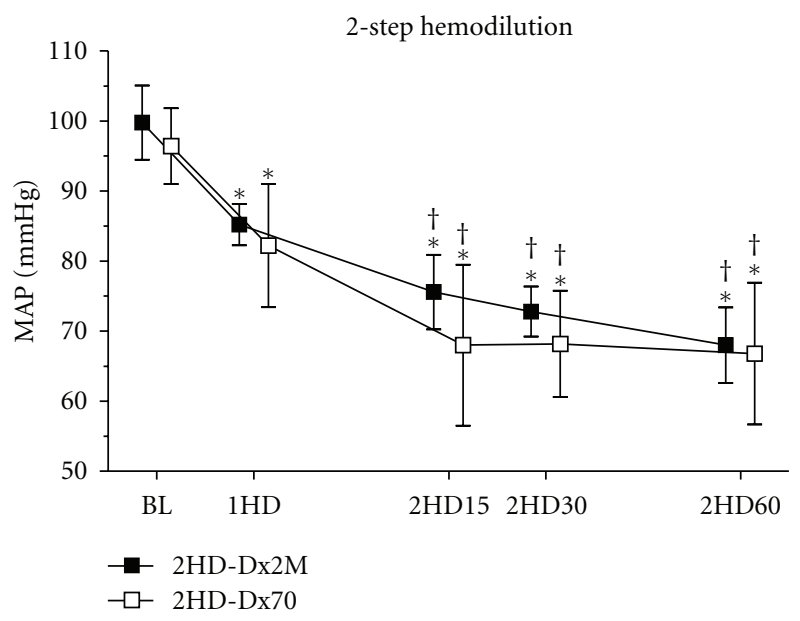

(b)

Figure 2: Mean arterial pressure (MAP) in the 1-step and 2step hemodilutions measured at baseline (BL), at 15, 30 and 60 minutes after hemodilution (HD15, HD30, HD60) and at first step hemodilution (1HD). Values are presented as means \pm SD. ${ }^{*} P<$ 0.05 compared to BL. ${ }^{\dagger} P<0.05$ compared to $1 \mathrm{HD}$.

the 1-step hemodilution to the 2-step hemodilution, CO decreased $9 \%$ and $12 \%$ in the group hemodiluted with Dx70 and Dx2M. In addition, the changes from the baseline in stroke volume (SV) and stroke work (SW) increased in the 1-step hemodilution while SV was maintained at the baseline and SW decreased relative to baseline in the 2-step hemodilution. An increased SW in the group hemodiluted with Dx2M indicates that the heart performs much work for higher whole blood and plasma viscosities.

Figure 5 illustrates examples of pressure-volume (PV) loops at baseline and after hemodilution. The PV loops in the 2-step hemodilution became smaller with particularly lower pressure compared to the PV loops at baseline and in the 1-step hemodilution. Furthermore, PV loops after hemodilution were shifted to the right of the baseline PV loop, indicating an increase of preload or end-diastolic volume. The area of PV loops corresponding to SW clearly 
TABLE 1: Hct, Hb, and rheological properties after complete hemodilution.

\begin{tabular}{|c|c|c|c|c|c|c|c|c|c|c|}
\hline \multirow{2}{*}{ Group } & \multicolumn{2}{|c|}{ Hct (\%) } & \multicolumn{2}{|c|}{$\mathrm{Hb}(\mathrm{g} / \mathrm{dL})$} & \multicolumn{2}{|c|}{ Blood viscosity (cP) } & \multicolumn{2}{|c|}{ Plasma viscosity $(\mathrm{cP})$} & \multicolumn{2}{|c|}{ Plasma COP $(\mathrm{mmHg})$} \\
\hline & $1 \mathrm{HD}$ & $2 \mathrm{HD}$ & $1 \mathrm{HD}$ & $2 \mathrm{HD}$ & $1 \mathrm{HD}$ & $2 \mathrm{HD}$ & $1 \mathrm{HD}$ & $2 \mathrm{HD}$ & $1 \mathrm{HD}$ & $2 \mathrm{HD}$ \\
\hline Blood (baseline) & & \pm 2 & 15.6 & \pm 0.7 & $4.7=$ & \pm 0.6 & $1.1=$ & \pm 0.1 & & \pm 2 \\
\hline $\mathrm{Dx} 2 \mathrm{M}$ & $27 \pm 2^{*}$ & $17 \pm 2^{*, \dagger, \ddagger}$ & $8.3 \pm 0.7^{*}$ & $5.2 \pm 0.8^{*, \dagger, \ddagger}$ & $3.6 \pm 0.2^{*, \ddagger}$ & $3.0 \pm 0.1^{*, \dagger, \ddagger}$ & $2.1 \pm 0.1^{*, \neq}$ & $2.0 \pm 0.1^{*, \neq}$ & $18 \pm 2$ & $17 \pm 4$ \\
\hline Dx70 & $27 \pm 2^{*}$ & $22 \pm 1^{*, \dagger}$ & $8.2 \pm 0.6^{*}$ & $6.7 \pm 0.5^{*, \dagger}$ & $3.1 \pm 0.1^{*}$ & $2.6 \pm 0.3^{*, \dagger}$ & $1.3 \pm 0.1^{*}$ & $1.4 \pm 0.1^{*}$ & $16 \pm 1$ & $17 \pm 2$ \\
\hline
\end{tabular}

The values of $\mathrm{Hct}, \mathrm{Hb}$ and rheological properties of blood and plasma at the baseline and after complete the 1-step hemodilution (1HD) and the 2-step hemodilution (2HD). Viscosity was measured at shear rate of $1601 / \mathrm{sec}$ at $37^{\circ} \mathrm{C}$. Values are means $\pm \mathrm{SD}$. ${ }^{*} P<0.05$ compared with baseline, ${ }^{\dagger} P<0.05$ compared with the 1 -step hemodilution using same plasma expander. ${ }^{\ddagger} P<0.05$ compared with Dx70 in the same step hemodilution.

TABLE 2: Cardiac function indices measured and derived from a pressure-volume (PV) measurement for the 1-step hemodilution and the 2-step hemodilution.

\begin{tabular}{|c|c|c|c|c|c|c|c|}
\hline \multirow{2}{*}{ Group } & \multirow{2}{*}{ Protocol } & \multicolumn{2}{|c|}{ Baseline } & \multicolumn{2}{|c|}{30 min after hemodilution } & \multicolumn{2}{|c|}{60 min after hemodilution } \\
\hline & & Dx70 & Dx2M & Dx70 & Dx2M & Dx70 & $\mathrm{Dx} 2 \mathrm{M}$ \\
\hline \multirow{2}{*}{$P_{\mathrm{es}}(\mathrm{mmHg})$} & $1 \mathrm{HD}$ & $122 \pm 5$ & $118 \pm 6$ & $106 \pm 12^{*}$ & $109 \pm 10$ & $105 \pm 8^{*}$ & $105 \pm 10^{*}$ \\
\hline & $2 \mathrm{HD}$ & $117 \pm 10$ & $120 \pm 7$ & $88 \pm 9^{*, \dagger}$ & $96 \pm 5^{*, \dagger}$ & $86 \pm 7^{*, \dagger}$ & $96 \pm 8^{*}$ \\
\hline \multirow{2}{*}{$P_{\text {ed }}(\mathrm{mmHg})$} & $1 \mathrm{HD}$ & $7 \pm 2$ & $8 \pm 4$ & $9 \pm 2$ & $8 \pm 2$ & $9 \pm 2$ & $8 \pm 2$ \\
\hline & $2 \mathrm{HD}$ & $5 \pm 2$ & $6 \pm 2$ & $7 \pm 3$ & $7 \pm 1$ & $6 \pm 2$ & $7 \pm 1$ \\
\hline \multirow{2}{*}{$\begin{array}{l}d P / d t_{\max } \\
(\mathrm{mmHg} / \mathrm{s})\end{array}$} & $1 \mathrm{HD}$ & $14,649 \pm 1,278$ & $12,810 \pm 1,194$ & $12,707 \pm 1,155^{*}$ & $11,832 \pm 2,404$ & $11,668 \pm 1,107^{*}$ & $12,045 \pm 1,460$ \\
\hline & $2 \mathrm{HD}$ & $14,060 \pm 1,245$ & $16,001 \pm 1,454$ & $10,054 \pm 1,117^{*, \dagger}$ & $11,194 \pm 1,084^{*}$ & $10,555 \pm 1,524^{*}$ & $10,631 \pm 1,460^{*}$ \\
\hline \multirow{2}{*}{$\begin{array}{l}d P / d t_{\min } \\
(\mathrm{mmHg} / \mathrm{s})\end{array}$} & $1 \mathrm{HD}$ & $-12,583 \pm 2,176$ & $-13,980 \pm 1,470$ & $-10,370 \pm 2,166$ & $-11,264 \pm 1,677^{*}$ & $-9,723 \pm 1,847$ & $-9,352 \pm 1,771^{*}$ \\
\hline & $2 \mathrm{HD}$ & $-12,689 \pm 3,151$ & $-13,846 \pm 1,525$ & $-8,683 \pm 1,535^{*}$ & $-9,242 \pm 1,870^{*}$ & $-8,474 \pm 1,805^{*, \dagger}$ & $-8,672 \pm 1,167^{*}$ \\
\hline \multirow{2}{*}{$\mathrm{CO}(\mathrm{mL} / \mathrm{min})$} & $1 \mathrm{HD}$ & $9 \pm 3$ & $10 \pm 2$ & $11 \pm 4$ & $12 \pm 3$ & $10 \pm 4$ & $13 \pm 3$ \\
\hline & $2 \mathrm{HD}$ & $9 \pm 1$ & $10 \pm 2$ & $9 \pm 1$ & $10 \pm 1$ & $9 \pm 1$ & $11 \pm 1^{\ddagger}$ \\
\hline \multirow{2}{*}{$\mathrm{SV}(\mu \mathrm{L})$} & $1 \mathrm{HD}$ & $18 \pm 7$ & $21 \pm 4$ & $22 \pm 9$ & $26 \pm 8$ & $22 \pm 8$ & $28 \pm 8$ \\
\hline & $2 \mathrm{HD}$ & $19 \pm 3$ & $21 \pm 4$ & $21 \pm 5$ & $23 \pm 5$ & $21 \pm 4$ & $24 \pm 4$ \\
\hline \multirow{2}{*}{$\begin{array}{l}\text { SW } \\
(\mathrm{mmHg} \cdot \mu \mathrm{L})\end{array}$} & $1 \mathrm{HD}$ & $2,020 \pm 691$ & $2,223 \pm 450$ & $2,257 \pm 863$ & $2,703 \pm 833$ & $2,181 \pm 781$ & $2,835 \pm 875$ \\
\hline & $2 \mathrm{HD}$ & $2,137 \pm 429$ & $2,280 \pm 388$ & $1,881 \pm 370$ & $2,183 \pm 404$ & $1,875 \pm 300$ & $2,254 \pm 312$ \\
\hline
\end{tabular}

Values are presented as means $\pm \mathrm{SD}$. $P_{\mathrm{es}}$, end systolic pressure; $P_{\text {ed }}$, end diastolic pressure; $d P / d t_{\max }$, maximum rate of pressure change; $d P / d t_{\min }$, minimum rate of pressure change; CO: cardiac output; SV: stroke volume; SW: stroke work. ${ }^{*} P<0.05$ compared with baseline. ${ }^{\dagger} P<0.05$ compared with 1 -step hemodilution using same plasma expander. ${ }^{\ddagger} P<0.05$ compared with Dx70 in the same step hemodilution.

demonstrated that Dx2M required higher work to pump out from the heart than Dx70. Both Dx70 and Dx2M significantly decreased systemic vascular resistance (SVR) compared to the baseline after hemodilution as shown in Figure $6(P<0.05)$.

\section{Discussion}

This study indicated that the reduction of oxygen-carrying capacity by the 2 -step hemodilution $(17 \% \mathrm{Hct})$ attenuated the cardiac performance and prevented the heart from handling the increase of plasma viscosity in our anesthetized model. The results in the 2-step hemodilution, as expected, showed the lower increments of stroke work, stroke volume, and cardiac output compared to the 1step hemodilution, representing the lower maintenance of oxygenation. Furthermore, during first 30 minutes after hemodilution, systolic indices such as $P_{\mathrm{es}}$ and $d P / d t_{\max }$ significantly decreased in the 2-step hemodilution compared with the 1-step hemodilution, especially hemodilution with Dx70.

At the Hct of $28 \%$, our results showed that an increase of plasma viscosity with Dx2M, a high viscosity plasma expander, demonstrated the effects on cardiac performance much better compared to Dx70, a low viscosity plasma expander, which resulted in the maintenance of $d P / d t_{\max }$ and the enhancement of cardiac output, stroke volume, and stroke work. This finding is in agreement with our previous study about cardiac mechanoenergetics using two different concentrations and viscosities of Dx2M in a moderate hemodilution $(28 \% \mathrm{Hct})$ [9]. Organ or tissue oxygenation is influenced by blood flow, oxygen-carrying capacity of blood, and oxygen extraction which are modulated when hemoglobin decreases [21]. In which present study, the ratio of oxygen delivery between the end of hemodilution $(28 \% \mathrm{Hct})$ and baseline $(52 \% \mathrm{Hct})$ was maintained near the baseline level due to an increase of cardiac output in a group hemodiluted with Dx2M. However, the reduction of the Hct to $17 \%$ while plasma viscosity increased $100 \%$ in anesthetized hamsters particularly decreased the oxygen delivery to the heart compared to the reduction of Hct to $28 \%$ even using Dx2M.

The reduction of oxygen-carrying capacity by hemodilution to $17 \% \mathrm{Hct}$ in an anesthetized hamster model demonstrated no significantly different effects of Dx2M on cardiac function compared to Dx70 although Dx2M has a higher viscosity than Dx70. Interestingly, the microcirculation studies 


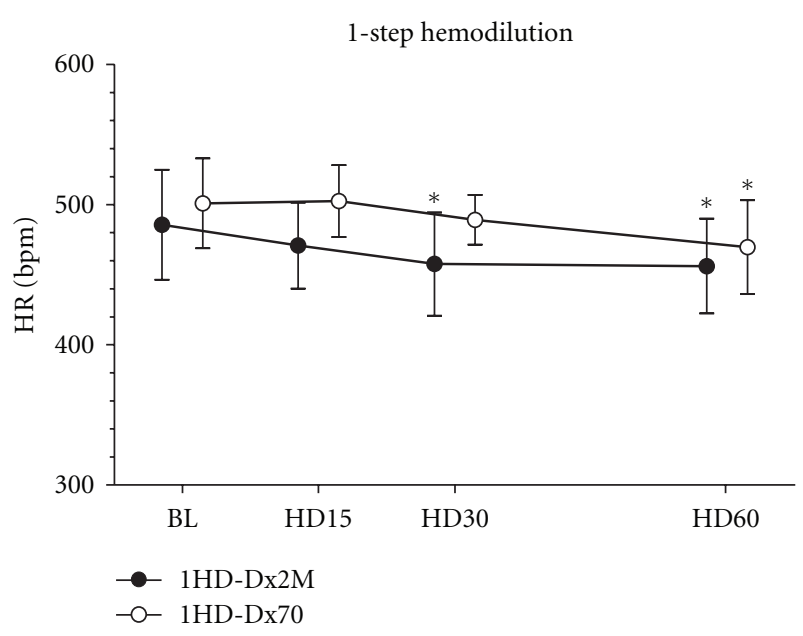

(a)

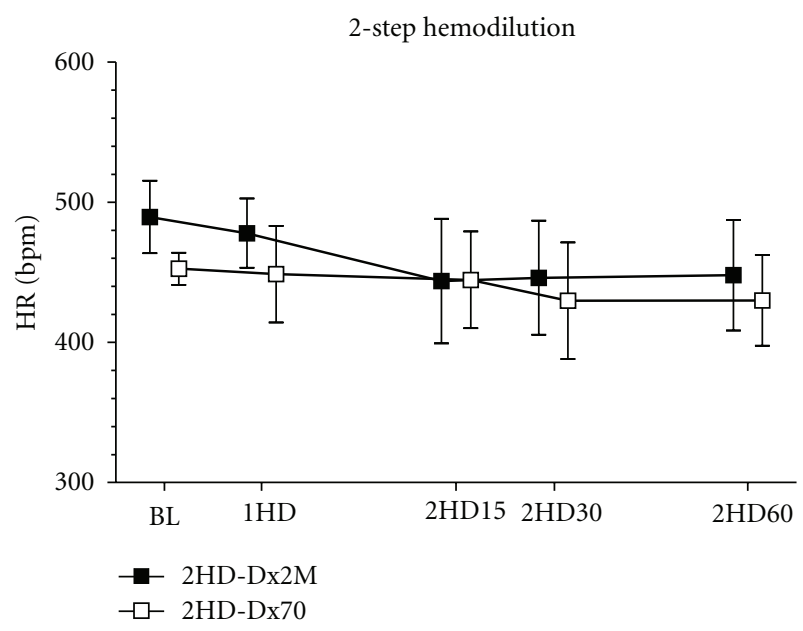

(b)

Figure 3: Heart rate (HR) in the 1-step and 2-step hemodilutions measured at baseline (BL), at 15, 30 and 60 minutes after hemodilution (HD15, HD30, HD60) and at first step hemodilution (1HD). Values are presented as means \pm SD. ${ }^{*} P<0.05$ compared to baseline.

in awake hamsters has demonstrated that increased plasma viscosity improved microvascular blood flow, functional capillary density, capillary pressure, cardiac output, and organ blood flow although the Hct was reduced to $\sim 10 \%$ $[3,6,7,22]$. The promising different results when the Hct is lowered to $17 \%$, comparing between the anesthetized and awake hamsters, might be the superimposition of the depressive effects of the anesthetic on the cardiovascular system. In addition, Hiebl et al. [23] performed the blood dilution study in anesthetized pigs and reported that the cardiac contraction markedly decreased when the Hct fell below $10 \%$ due to a significant reduction in left ventricular oxygen partial pressure. According to their data, there were no significant differences in oxygen partial pressure and systemic blood pressure when the Hct was reduced from $20 \%$ to $15 \%$. Acute hemodilution to the Hct of $25 \%$ using a foxhound model caused an increased cardiac index and

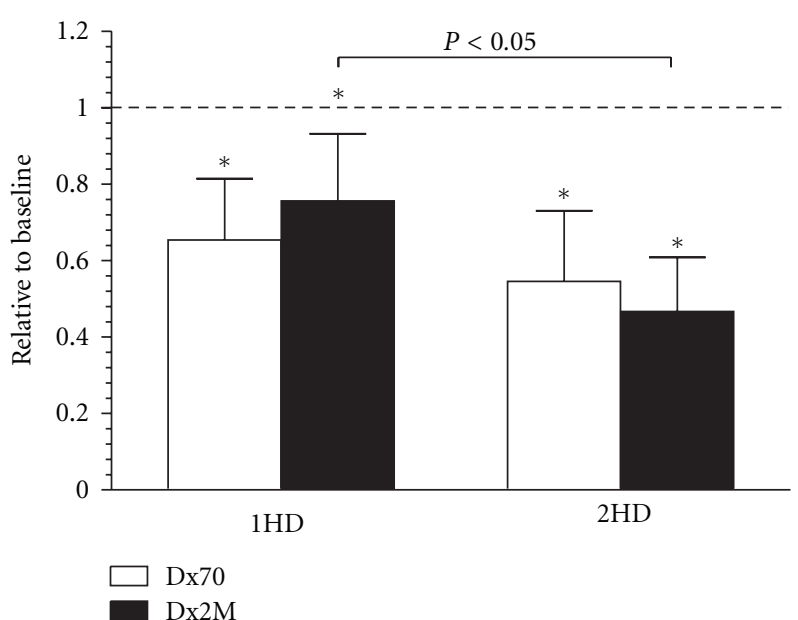

FIGURE 4: Oxygen delivery relative to baseline in the 1-step and 2step hemodilution. Values are presented as means \pm SD. Dash line represents the baseline. ${ }^{*} P<0.05$ compared to baseline.

oxygen extraction ratio compared to the baseline, whereas oxygen tension significantly decreased when the Hct is lowered beyond 25\% [24]. Tissue oxygenation increased after hemodilution until the Hct was lower than $25 \%$ and then the tissue oxygenation gradually decreased. Since the brain and myocardium have higher oxygen demand, the matching of oxygen delivery to tissue oxygenation is mandatory to maintain vital organ function. It has been found that oxygencarrying capacity is adequately supplied to the myocardium down to an Hct of $25 \%$ [25]. However, these studies partially support our results that a lower Hct by the 2-step hemodilution decreased the cardiac activity which required oxygen, as indicated by lower stroke work.

Suga and colleagues [26-29] demonstrated that oxygen consumption is proportional to the systolic pressurevolume area, which is equal to the combination of external mechanical work (PV loop area) and end-systolic elastic potential energy. Our study showed that the PV loop area in a group treated with $\mathrm{Dx} 2 \mathrm{M}$ was higher than that in a group hemodiluted with Dx70, implying higher oxygen demand to pump out blood diluted with Dx2M from the heart. Therefore, the reduction of oxygen delivery by hemodilution with a high viscosity plasma expander raises a concern about the critical point of the hematocrit and cardiac oxygenation, especially under a state of anesthesia.

Many studies have demonstrated that animals in a state of anesthesia have depressive cardiac function $[1,13,14,17,30]$. Dosages and types of anesthetics particularly affect hemodynamic parameters and indices of systolic and diastolic function. Therefore, in a state of anesthesia combined with an anemic condition, the heart might operate with lower efficiency [31]. This could lead to the deterioration of the effects of an increase in plasma viscosity in cases where blood dilution reduces the Hct to a level lower than $28 \%$.

Blood viscosity is a determinant of resistance to blood flow. Lowering blood viscosity by a reduction of red blood cells causes a decrease in outflow impedance or afterload 


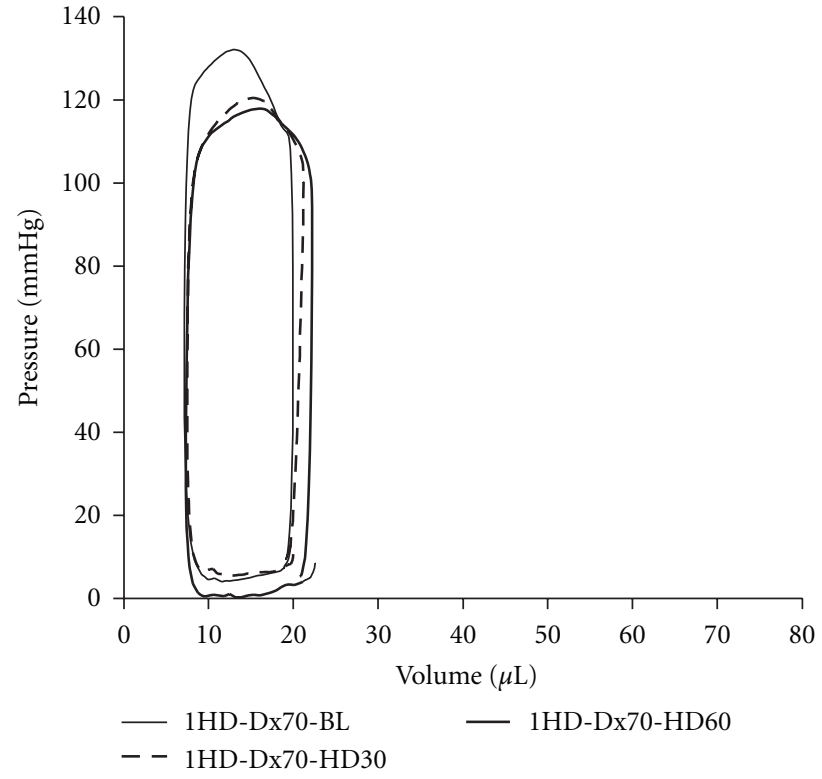

(a)

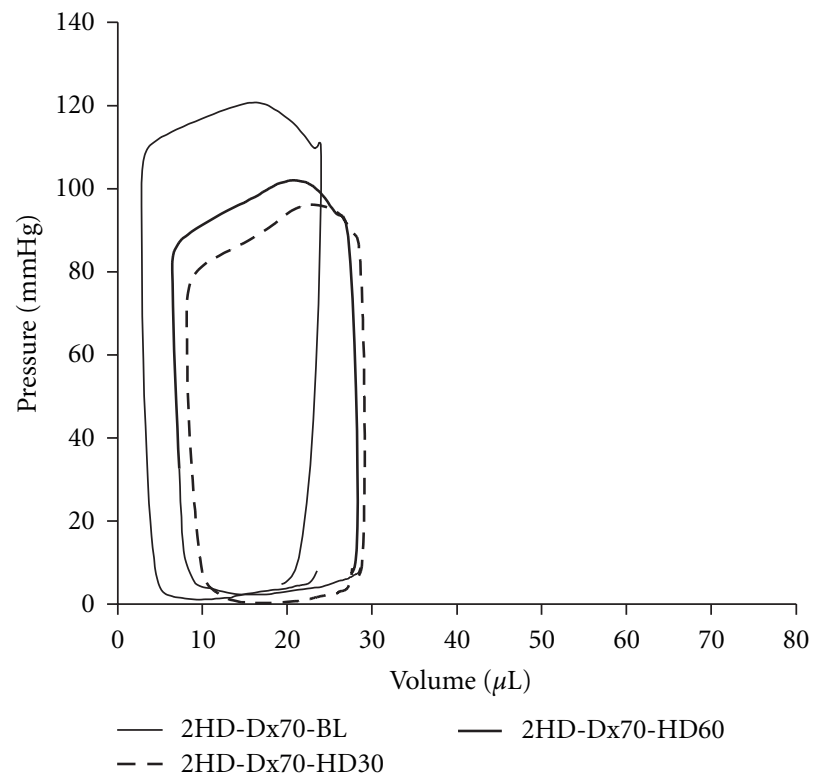

(c)

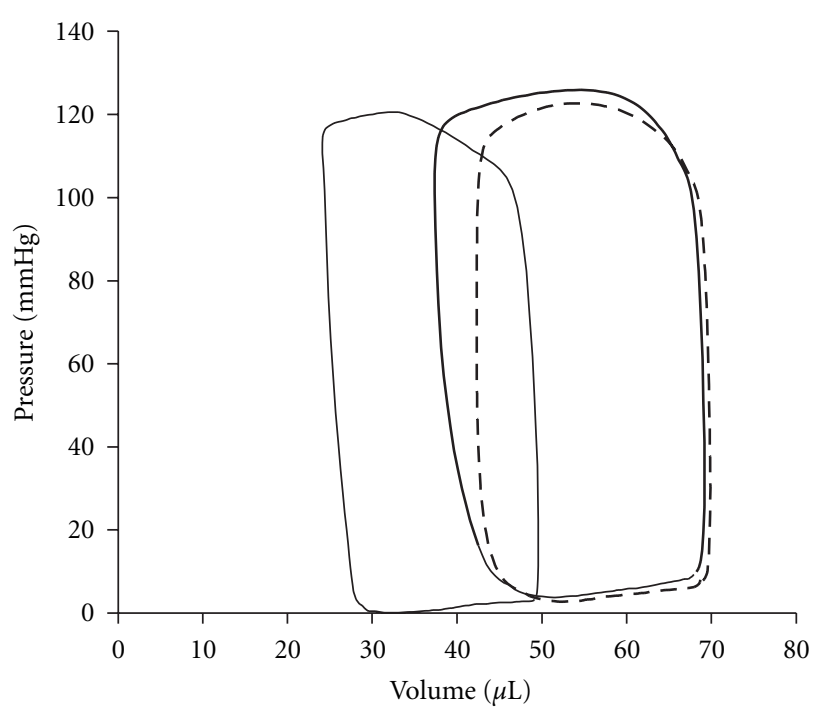

1HD-Dx2M-BL $\quad$ 1HD-Dx2M-HD60

- - 1HD-Dx2M-HD30

(b)

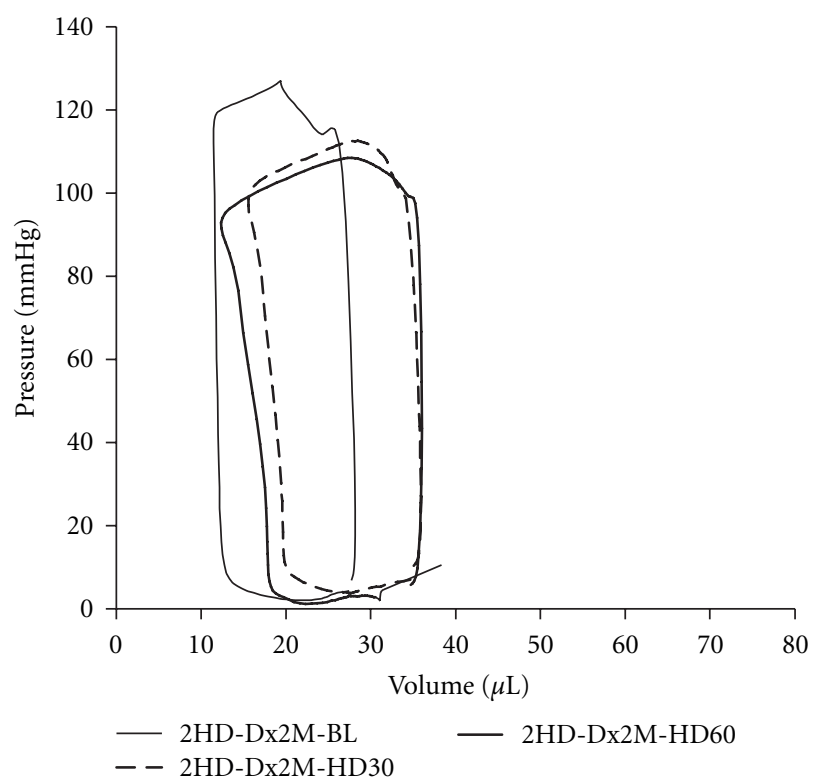

(d)

FIGURE 5: Pressure-volume loops at the baseline (BL), at 30 minutes (HD30) and 60 minutes (HD60) after the 1-step hemodilution (1HD) and 2-step hemodilution (2HD).

suggesting increased cardiac output and decreased MAP after hemodilution [32]. Similarly, our findings in systemic vascular resistance decreased after hemodilution while stroke volume increased, implying an increased preload due to the Frank-Starling law of the heart. However, systemic vascular resistance calculated in our study did not show a significant difference between a group hemodiluted with Dx2M and a group treated with Dx70 in both protocols. Therefore, in the 1-step and 2-step hemodilutions, the higher cardiac output in a group hemodiluted with Dx2M might be affected by cardiac contractility and preload, not afterload.
In conclusion, increasing plasma viscosity associated with lowering oxygen-carrying capacity should be considerably balanced to maintain the cardiac performance, especially in the state of anesthesia. Our findings suggest that, although plasma viscosity in anesthetized animals increased, a reduction of the hematocrit from $28 \%$ to $17 \%$ lowered oxygen delivery and attenuated cardiac performance. Therefore, the level of a critical hematocrit to match the effects of increasing the plasma viscosity and making the whole blood viscosity closed to the physiological value is important to determine. 


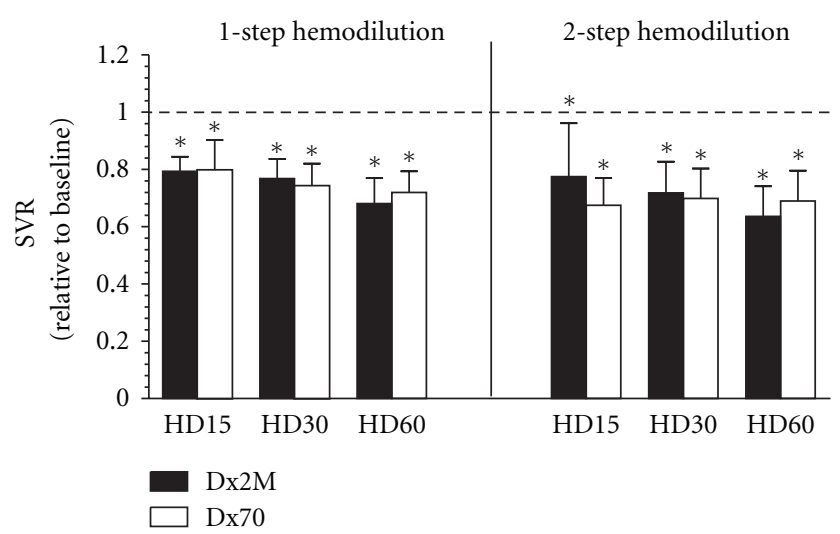

FIGURE 6: Systemic vascular resistance (SVR) relative to baseline in the 1-step and 2-step hemodilutions measured at 15, 30 and 60 minutes after hemodilution (HD15, HD30, HD60). Values are presented as means $\pm \mathrm{SD}$. Dash line represents the baseline. ${ }^{*} P<$ 0.05 compared to baseline.

\section{Acknowledgments}

The authors greatly thank Cynthia Walser for the surgical preparation of the animals. This work was supported by the Bioengineering Research Partnership grant R24-HL64395, Program project P01-HL071064, grant R01-HL62354, R01HL62318, and the Faculty of Medicine, Prince of Songkla University grant 54-096-25-2-3.

\section{References}

[1] O. P. Habler, M. S. Kleen, A. H. Podtschaske et al., "The effect of acute normovolemic hemodilution (ANH) on myocardial contractility in anesthetized dogs," Anesthesia and Analgesia, vol. 83, no. 3, pp. 451-458, 1996.

[2] G. J. Crystal, M. W. Rooney, and M. R. Salem, "Myocardial blood flow and oxygen consumption during isovolemic hemodilution alone and in combination with adenosineinduced controlled hypotension," Anesthesia and Analgesia, vol. 67, no. 6, pp. 539-547, 1988.

[3] A. G. Tsai and M. Intaglietta, "High viscosity plasma expanders: volume restitution fluids for lowering the transfusion trigger," Biorheology, vol. 38, no. 2-3, pp. 229-237, 2001.

[4] P. Cabrales, A. G. Tsai, and M. Intaglietta, "Is resuscitation from hemorrhagic shock limited by blood oxygen-carrying capacity or blood viscosity?" Shock, vol. 27, no. 4, pp. 380-389, 2007.

[5] P. Cabrales, A. G. Tsai, and M. Intaglietta, "Microvascular pressure and functional capillary density in extreme hemodilution with low-and high-viscosity dextran and a low-viscosity Hb-based O2 carrier," American Journal of Physiology, vol. 287, no. 1, pp. H363-H373, 2004.

[6] P. Cabrales, J. Martini, M. Intaglietta, and A. G. Tsai, "Blood viscosity maintains microvascular conditions during normovolemic anemia independent of blood oxygen-carrying capacity," American Journal of Physiology, vol. 291, no. 2, pp. H581-H590, 2006.

[7] P. Cabrales, A. G. Tsai, and M. Intaglietta, "Alginate plasma expander maintains perfusion and plasma viscosity during extreme hemodilution," American Journal of Physiology, vol. 288, no. 4, pp. H1708-H1716, 2005.
[8] C. De Wit, C. Schäfer, P. Von Bismarck, S. S. Bolz, and U. Pohl, "Elevation of plasma viscosity induces sustained NOmediated dilation in the hamster cremaster microcirculation in vivo," Pflugers Archiv European Journal of Physiology, vol. 434, no. 4, pp. 354-361, 1997.

[9] S. Chatpun and P. Cabrales, "Cardiac mechanoenergetic cost of elevated plasma viscosity after moderate hemodilution," Biorheology, vol. 47, no. 3-4, pp. 225-237, 2010.

[10] S. Chatpun and P. Cabrales, "Effects of plasma viscosity modulation on cardiac function during moderate hemodilution," Asian Journal of Transfusion Science, vol. 4, no. 2, pp. 102-108, 2010.

[11] W. W. Muir III, A. Kijtawornrat, Y. Ueyama, S. V. Radecki, and R. L. Hamlin, "Effects of intravenous administration of lactated Ringer's solution on hematologic, serum biochemical, rheological, hemodynamic, and renal measurements in healthy isoflurane-anesthetized dogs," Journal of American Veterinary Medical Association, vol. 239, no. 5, pp. 630-637, 2011.

[12] G. J. Crystal and M. R. Salem, "Myocardial and systemic hemodynamics during isovolemic hemodilution alone and combined with nitroprusside-induced controlled hypotension," Anesthesia and Analgesia, vol. 72, no. 2, pp. 227-237, 1991.

[13] O. Fraga Ade, D. T. Fantoni, D. A. Otsuki, C. A. Pasqualucci, M. C. D. Abduch, and J. O. Costa Auler, "Evidence for myocardial defects under extreme acute normovolemic hemodilution with hydroxyethyl starch and lactated ringer's solution," Shock, vol. 24, no. 4, pp. 388-395, 2005.

[14] B. E. Ickx, M. Rigolet, and P. J. Van Der Linden, "Cardiovascular and metabolic response to acute normovolemic anemia: effects of anesthesia," Anesthesiology, vol. 93, no. 4, pp. 10111016, 2000.

[15] A. S. Most, N. A. Ruocco Jr., and H. Gewirtz, "Effect of a reduction in blood viscosity on maximal myocardial oxygen delivery distal to a moderate coronary stenosis," Circulation, vol. 74, no. 5, pp. 1085-1092, 1986.

[16] K. D. Vandegriff, R. J. Rohlfs, M. D. Magde, and R. M. Winslow, "Hemoglobin-oxygen equilibrium curves measured during enzymatic oxygen consumption," Analytical Biochemistry, vol. 256, no. 1, pp. 107-116, 1998.

[17] P. Pacher, T. Nagayama, P. Mukhopadhyay, S. Bátkai, and D. A. Kass, "Measurement of cardiac function using pressurevolume conductance catheter technique in mice and rats," Nature Protocols, vol. 3, no. 9, pp. 1422-1434, 2008.

[18] J. Baan, E. T. Van der Velde, and H. G. De Bruin, "Continuous measurement of left ventricular volume in animals and humans by conductance catheter," Circulation, vol. 70 , no. 5 , pp. 812-823, 1984.

[19] D. Georgakopoulos, W. A. Mitzner, C. H. Chen et al., "In vivo murine left ventricular pressure-volume relations by miniaturized conductance micromanometry," American Journal of Physiology, vol. 274, no. 4, pp. H1416-H1422, 1998.

[20] P. Steendijk, E. T. Van der Velde, and J. Baan, "Left ventricular stroke volume by single and dual excitation of conductance catheter in dogs," American Journal of Physiology, vol. 264, no. 6, pp. H2198-H2207, 1993.

[21] F. Metivier, S. J. Marchais, A. P. Guerin, B. Pannier, and G. M. London, "Pathophysiology of anaemia: focus on the heart and blood vessels," Nephrology Dialysis Transplantation, vol. 15, supplement 3, no. 3, pp. 14-18, 2000.

[22] P. Cabrales and A. G. Tsai, "Plasma viscosity regulates systemic and microvascular perfusion during acute extreme anemic 
conditions," American Journal of Physiology, vol. 291, no. 5, pp. H2445-H2452, 2006.

[23] B. Hiebl, C. Mrowietz, K. Ploetze, K. Matschke, and F. Jung, "Critical hematocrit and oxygen partial pressure in the beating heart of pigs," Microvascular Research, vol. 80, no. 3, pp. 389393, 2010.

[24] M. Freitag, T. Standl, E. P. Horn, S. Wilhelm, and J. Schulte Am Esch, "Acute normovolaemic haemodilution beyond a haematocrit of 25\%: ratio of skeletal muscle tissue oxygen tension and cardiac index is not maintained in the healthy dog," European Journal of Anaesthesiology, vol. 19, no. 7, pp. 487-494, 2002.

[25] D. R. Spahn, B. J. Leone, J. G. Reves, and T. Pasch, "Cardiovascular and coronary physiology of acute isovolemic hemodilution: a review of nonoxygen-carrying and oxygencarrying solutions," Anesthesia and Analgesia, vol. 78, no. 5, pp. 1000-1021, 1994.

[26] H. Suga, Y. Igarashi, O. Yamada, and Y. Goto, "Cardiac oxygen consumption and systolic pressure volume area," Basic Research in Cardiology, vol. 81, supplement 1, no. 1, pp. 39-50, 1986.

[27] H. Suga and G. Matsushita, "Real time analog computation of left ventricular systolic pressure volume area as predictor of oxygen consumption," Japanese Heart Journal, vol. 22, no. 6, pp. 997-1003, 1981.

[28] H. Suga, T. Hayashi, and M. Shirahata, "Ventricular systolic pressure-volume area as predictor of cardiac oxygen consumption," American Journal of Physiology, vol. 240, no. 1, pp. H39H44, 1981.

[29] H. Suga, T. Hayashi, and S. Suehiro, "Equal oxygen consumption rates of isovolumic and ejecting contractions with equal systolic pressure-volume areas in canine left ventricle," Circulation Research, vol. 49, no. 5, pp. 1082-1091, 1981.

[30] M. Zorniak, K. Mitrega, S. Bialka, M. Porc, and T. F. Krzeminski, "Comparison of thiopental, urethane, and pentobarbital in the study of experimental cardiology in rats in vivo," Journal of Cardiovascular Pharmacology, vol. 56, no. 1, pp. 38-44, 2010.

[31] G. J. Crystal, "Myocardial oxygen supply-demand relations during isovolemic hemodilution," Advances in Pharmacology, vol. 31, pp. 285-312, 1994.

[32] K. Messmer, U. Kreimeier, and M. Intaglietta, "Present state of intentional hemodilution," European Surgical Research, vol. 18, no. 3-4, pp. 254-263, 1986. 


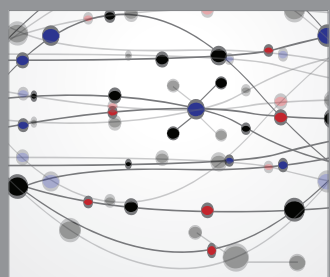

The Scientific World Journal
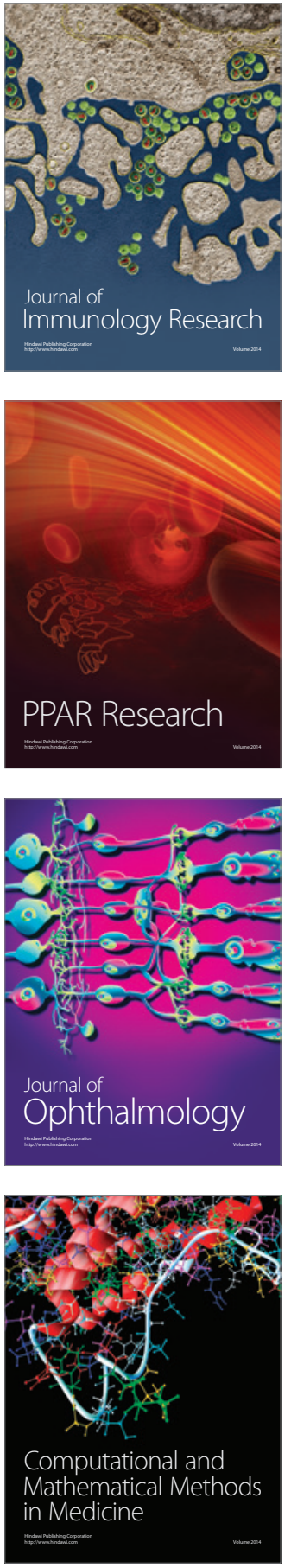

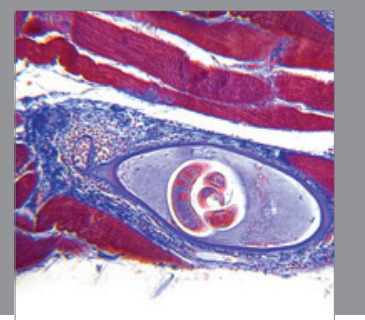

Gastroenterology

Research and Practice
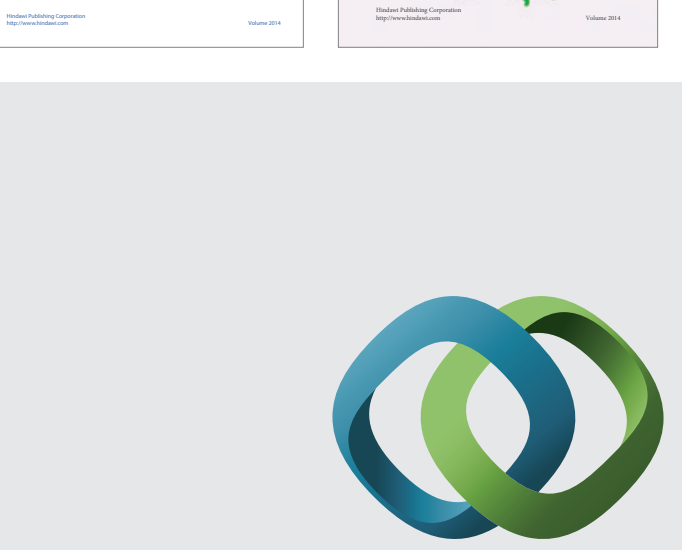

\section{Hindawi}

Submit your manuscripts at

http://www.hindawi.com
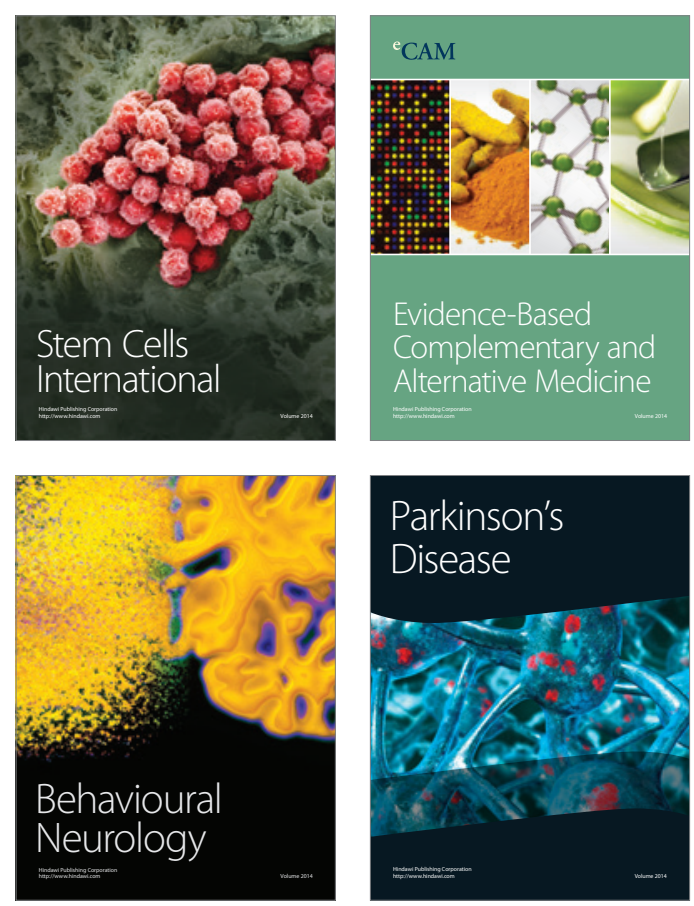

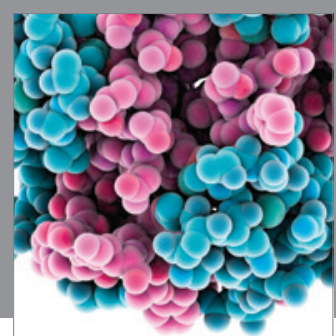

Journal of
Diabetes Research

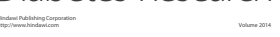

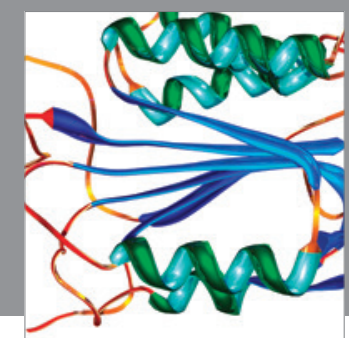

Disease Markers
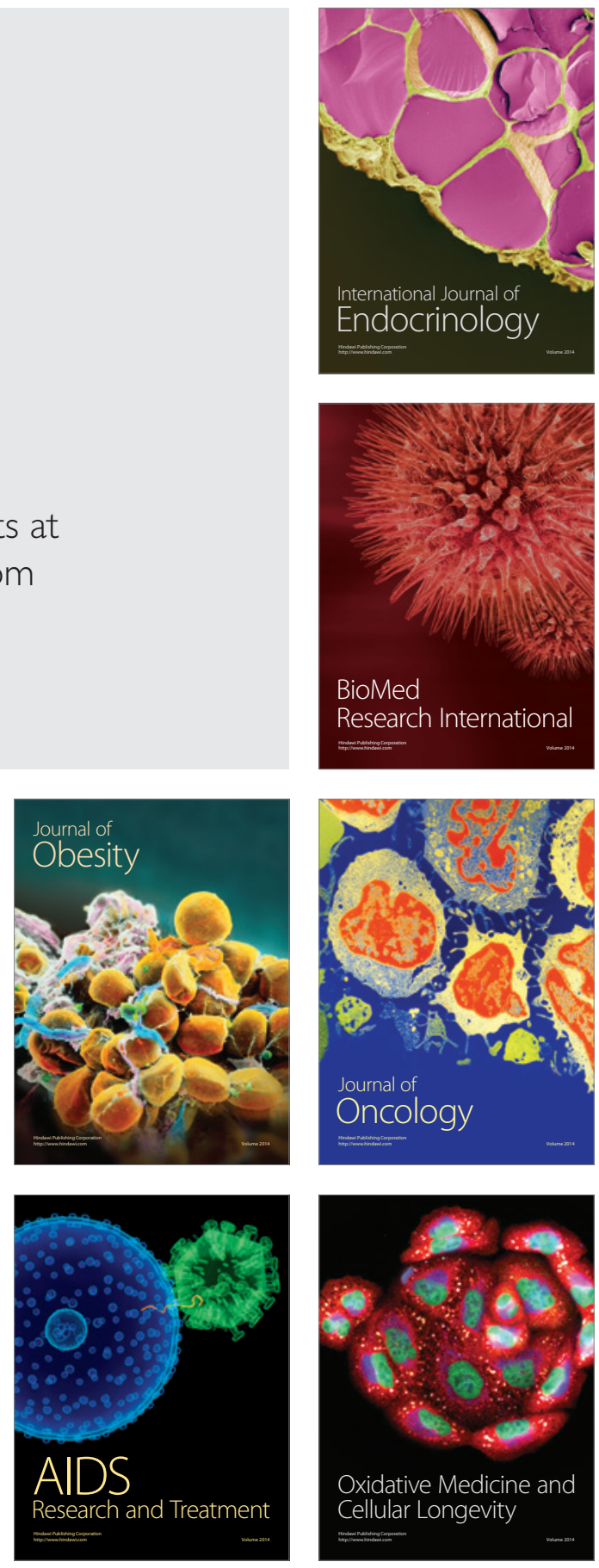